\title{
Study the Effects of Exposing the Skin and Eyes of Mice to Ultraviolet-C Radiation
}

\author{
Abu Bakr El-Bediwi ${ }^{1}$, El-Sayed Kamel Areida ${ }^{2}$, Eman Eid \\ ${ }^{1}$ Physics Department, Faculty of Science, Mansoura University, Egypt \\ ${ }^{2}$ Zoology Dept., Faculty of Science, Mansoura University, Egypt
}

\begin{abstract}
The study was carried out to investigate the harmful effects of UVC radiation on skin and eye of mice. Eighty male Albino mice were used in this study. The mice were equally divided into three groups as control, UVC and recovery. UVC group mice were divided into two groups first irradiated for lhour per day and second irradiated 2 hour per day during 30 days. Autopsy samples were taken from the skin and eyes of mice in different groups and fixed in 10\% formol saline for twenty four hours. The obtained tissue sections were collected on glass slides, deparaffinized and stained by hematoxylin and eosin stain for examination through the light electric microscope. The results showed that, UVC radiation has harmful effects on skin and eye of mice and the recovery was found insignificant.
\end{abstract}

Keywords: UVC radiation, skin, eye, mice, recovery

\section{INTRODUCTION}

Ultraviolet radiation (UVR) occupies that portion of the electromagnetic spectrum from at least 100 to 400 nanometers as shown in Figure (1). In discussing UVR biological effects, the International Commission on Illumination has divided the UV spectrum into three bands. The band $315-400 \mathrm{~nm}$ is designated as UVA, 280 to $315 \mathrm{~nm}$ as UVB and 100 to $280 \mathrm{~nm}$ as UVC. Wavelengths below $180 \mathrm{~nm}$ are of little practical biologic significance since they are readily absorbed in air [1]. UVR is used in a wide variety of medical and industrial processes and for cosmetic purposes. These include photo curing of inks and plastics (UVA and UVB), photo resist processes (all UV), solar simulation (all UV), cosmetic tanning (UVA and UVB), fade testing (UVA and UVB), dermatology (all UV), and dentistry (UVA). Even though the principal operating wavelengths for most of these processes are in the UVA, almost always some shorter wavelength (UVB and UVC) radiation and violet light are emitted as well. Many industrial applications employ arc sources for heat or light, which also produce UVR as an unwanted admixture for which control measures may be necessary. While it is generally agreed that some low level exposure to UVR benefits health, there are adverse effects that necessitate the development and use of exposure limits (EL) for UVR. However, the development of UVR EL poses a real challenge to achieve a realistic balance between beneficial and adverse health effects. The primary organs of the body affected by UV light hazards are the skin and the eyes. An unfortunate property of UV radiation is that there are no immediate warning symptoms to indicate overexposure until well after the exposure has occurred. Symptoms of overexposure include varying degrees of erythema (sunburn) or photokeratitis typically do not appear until hours after the exposure [2].The cornea and lens are the main areas of the eyes affected by UV radiation. Various components of the human eye are susceptible to damage arising from photochemical effects as a result of extended exposure to direct/reflected UV radiation. UV radiation exposure can injure the cornea, the outer protective coating of the eye. Photokeratitis is a painful inflammation of the eye caused by UV radiation-induced lesions on the cornea. Symptoms include a sensation of sand in the eye that may last up to two days. This condition usually does not present until 6 to 12 hours following the UV exposure. Although very painful (often described as having sand in the eyes), this condition is usually temporary (a few days) because in most cases the corneal cells will grow back. Chronic exposures to acute high-energy UV radiation can lead to the formation of cataracts. UV radiation can initiate a photochemical reaction called erythema within exposed skin. This "sunburn" can be quite severe and can occur as a result of only a few seconds exposure. Effects are exaggerated for skin photosensitized by agents such as coal tar product found in anti-dandruff shampoos, certain foods, certain medications. Chronic skin exposure to UV radiation has been linked to premature skin aging, wrinkles and skin cancer. 


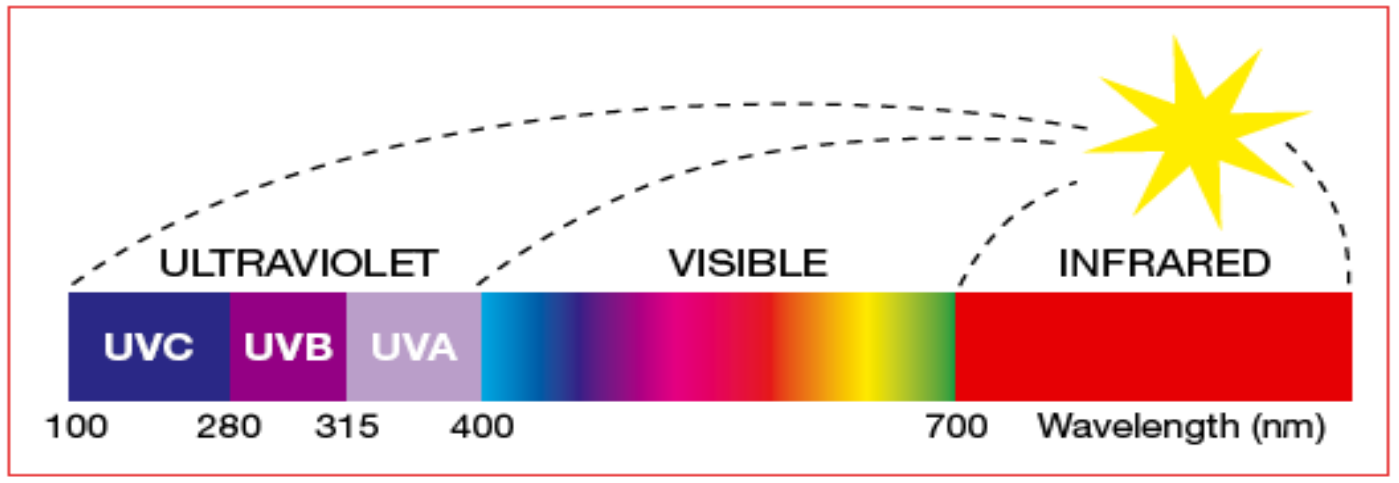

Figure1. The light spectrum [3]

The watt $(\mathrm{W})$, the fundamental unit of optical power, is defined as a rate of energy of one joule (J) per second [4]. Optical power is a function of both the number of photons and the wavelength. Each photon carries an energy that is described by Planck's equation, $\mathrm{Q}=\mathrm{h} \mathrm{c} / \lambda$ where $\mathrm{Q}$ is the photon energy (joules), $h$ is Planck's constant $\left(6.623 \times 10^{-34} \mathrm{~J} \mathrm{~s}\right), \mathrm{c}$ is the speed of light $\left(2.998 \times 108 \mathrm{~m} \mathrm{~s}^{-1}\right)$ and $\lambda$ is the wavelength of radiation (meters). All light measurement units are spectral, spatial, or temporal distributions of optical energy. Figure (2) shows short wavelength ultraviolet light has much more energy per photon than either visible or long wavelength infrared.

Mathematically, UV dosage can be expressed as a product of intensity and exposure (residence) time. The most commonly used units of UV dosage are microwatt-seconds per square centimetre $(\mu \mathrm{W}$ $\mathrm{Sec} / \mathrm{Cm}^{2}$ ). The aim of this work was to study the effect of ultraviolet $\mathrm{C}$ radiation on the skin and eyes of mice and the recovery possibility.

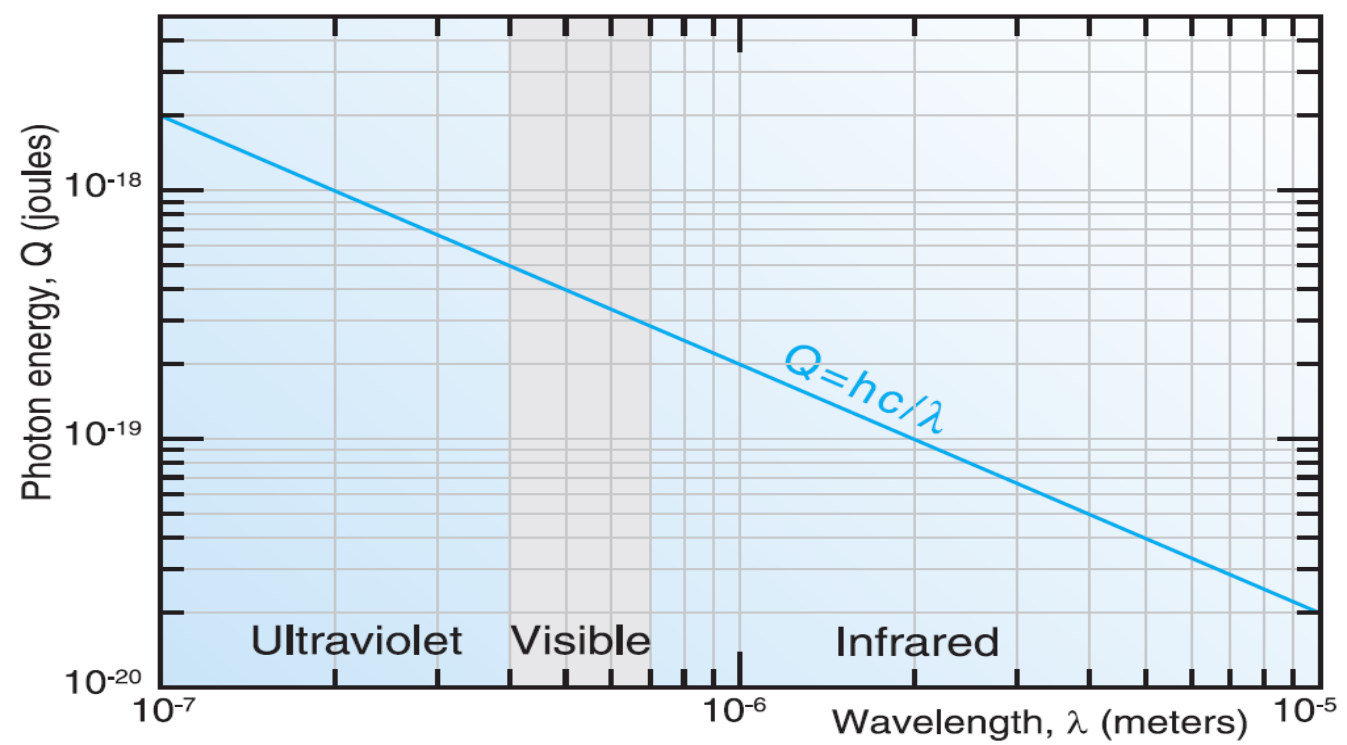

Figure2. Planck's equation shows photon energy vs. wavelength [4]

\section{MATERIALS AND MethodS}

\subsection{Animals}

A total of 80 male mice with weighing 20-25 g were used in this study and divided into four main groups:

1- Control mice group (A): Normal animals.

2- UVC-irradiated mice group (B \& C):

B: Animals constantly exposed to UVC radiation (1hour daily) for one month.

C: Animals constantly exposed to UVC-radiation (2hour daily) for one month.

3- Recovery group (D): Recovery animals after constantly exposer to UVC radiation (2 hour daily) for one month. 


\subsection{Irradiation Facilities}

The mice were placed in a special wood box $(60 \times 60 \times 60 \mathrm{~cm})$ and many small holes were made in the box sides to enable the mice to alive during the experiment of irradiation.

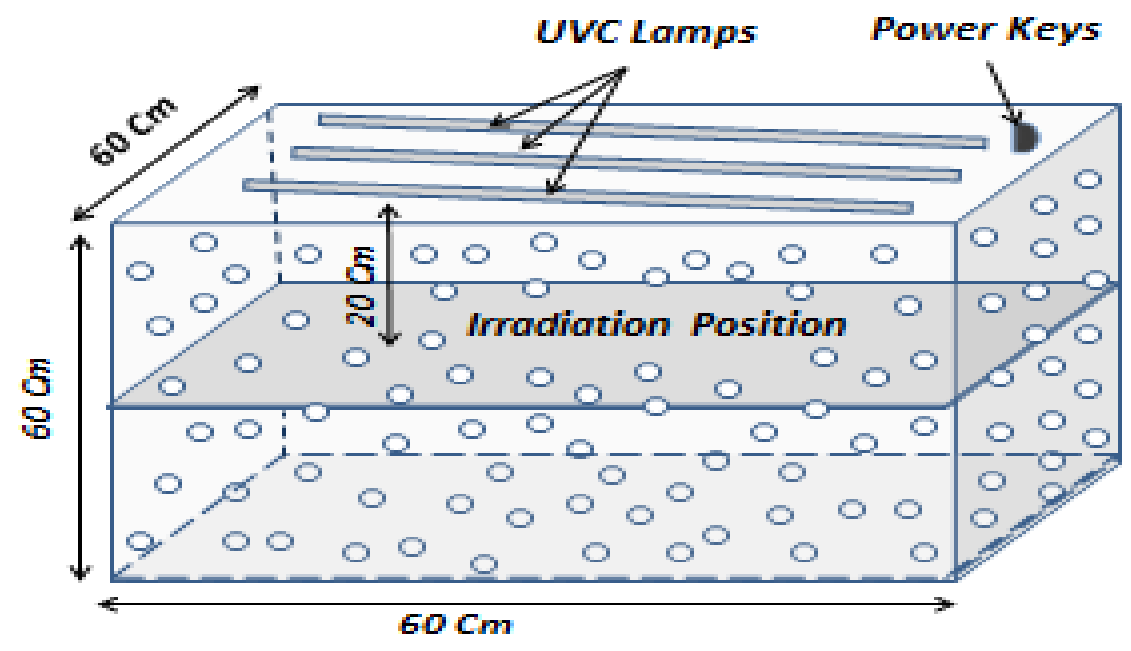

Figure3. The special wood box, where the mice were exposed to UVC radiation

\subsection{UV-C Source}

Three "Sylvania G15W" ultraviolet lamps in 15 Watt powers for each one and in $45 \mathrm{~cm}$ length were placed to the internal top surface of the wood box. These lamps consist of a tubular glass envelope and emit more than $85 \%$ of their energy in the UV-C ultra violet radiation with a peak at $253.7 \mathrm{~nm}$ for germicidal action [6]. Shape, electrical characteristics and lighting circuits are similar to general fluorescent lamps. The majority of germicidal lamps operate most efficiently in still air at an ambient temperature of $25^{\circ}$. All lamps are ozone free. A protective coating on the inside of the lamp limits the depreciation of the UV-C output.

Experiments were performed on mice at the animal house of Biophysics Department, Faculty of Science, Mansoura University, Egypt, under conventional laboratory conditions. All animals in control and experimental group were housed collectively in polycarbonate cages 30x40x40 cm (W x $\mathrm{L} \times \mathrm{H}$ ) and given access to standard laboratory food and water. Mice were classified into four groups: control and three UVR-treated groups. Mice were exposed to UVC radiation 1hour daily (group B)and 2hour daily (group C \& D)for one month. The dose delivered to the mice were calculated and adjusted at $20 \mathrm{~cm}$ from the lamps in the middle of the irradiation shell in order to be sure that all the mice receive a uniform and homogenous field of irradiation. A timer was used to standardize UVC exposure times.

\subsection{Method}

Following the end of exposure and post exposure periods autopsy samples were taken from the skin and eyes of mice in different groups and fixed in $10 \%$ formol saline for twenty four hours. Washing was done in tap water then serial dilutions of alcohol (methyl, ethyl and absolute ethyl) were used for dehydration. Specimens were cleared in xylene and embedded in paraffin at 56 degree in hot air oven for twenty four hours. Paraffin bees wax tissue blocks were prepared for sectioning at 4 microns thickness by slidge microtome. The obtained tissue sections were collected on glass slides, deparaffinized and stained by hematoxylin and eosin stain for examination through the light electric microscope [5].

\section{RESULTS AND DISCUSSION}

\subsection{Histopathological Findings}

3.1.1. Skin

Group (A): (mice kept as control)

There was no hiostopathological alteration and the normal histological structure of the epidermis and the underlying dermis were recorded in Figure (4). 

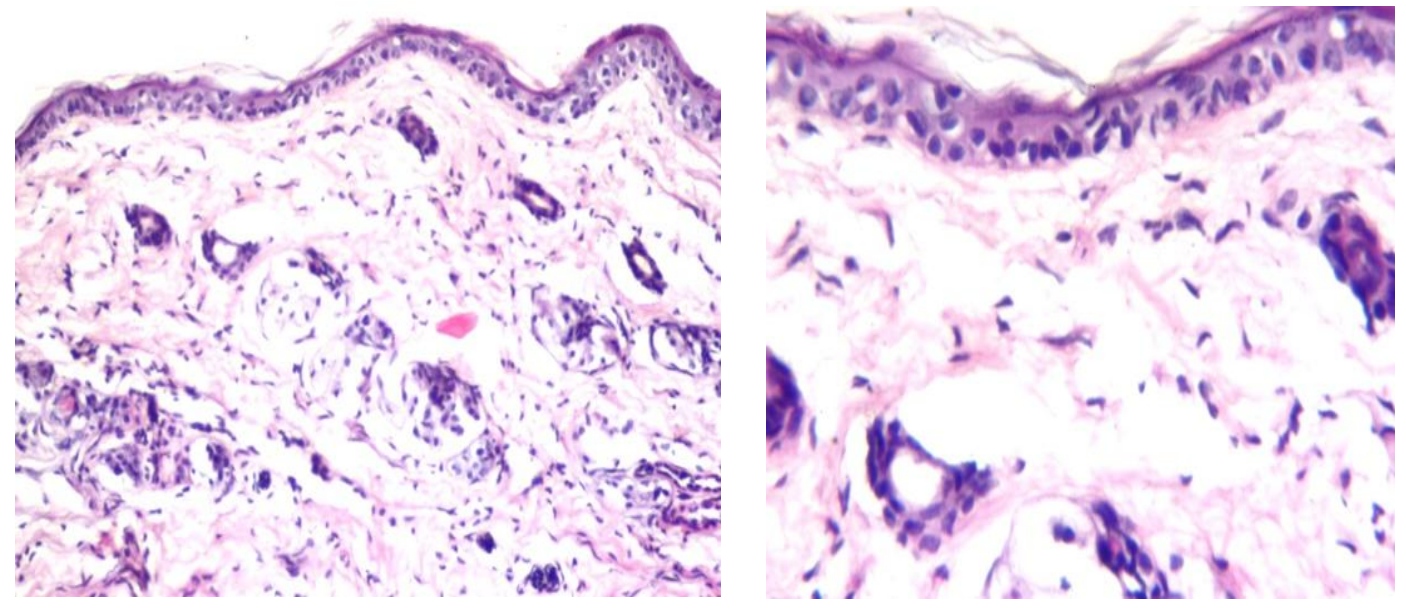

Figure4. Samples taken from the skin of mice kept as control

Group (B): (mice exposed to UV-C for one hour)

The different layers of the epidermis were losing while the basal cell layer still intact as shown in Figure (5).
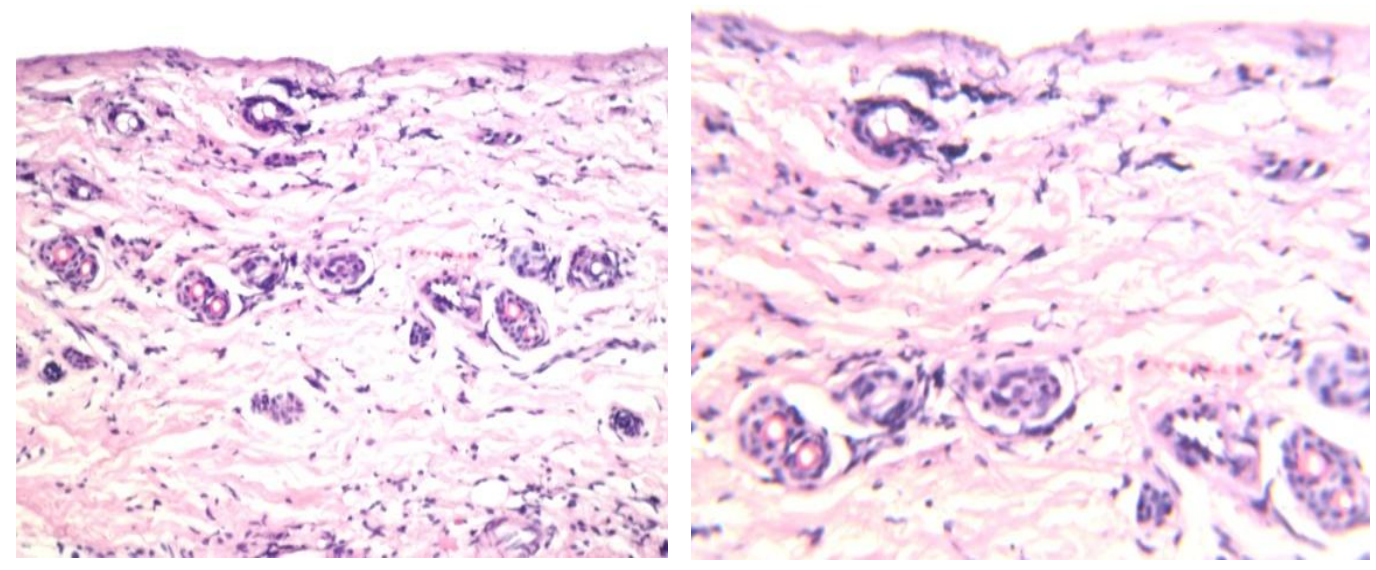

Figure5. Samples taken from the skin of mice exposed to UV-C for one hour

Group (C): (mice exposed to UV-C for two hour)

There was loss of the epidermal layers with intact basement membrane associated with invagination into the dermisas shown in Figure (6).
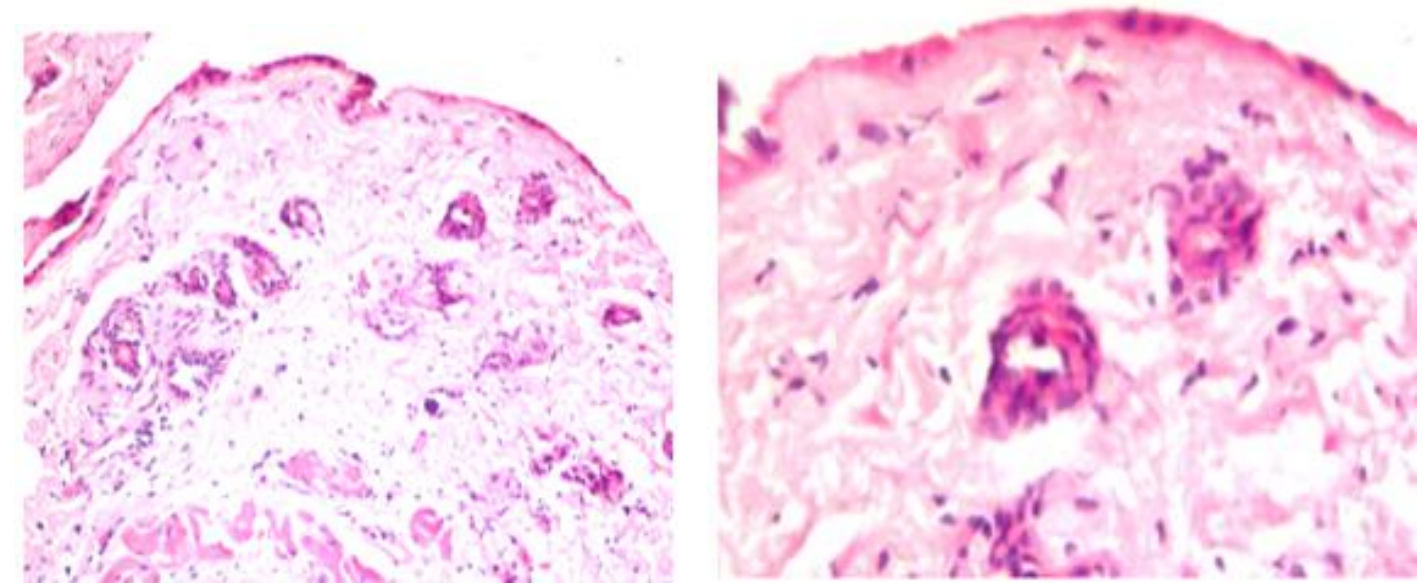

Figure6. Samples taken from the skin of mice exposed to UV-C for two hour

Group (D): (Recovery mice after exposer to UV-C for two hour)

The epidermal layer and the underlying dermis were intact while the invagination still present as shown in Figure (7). 

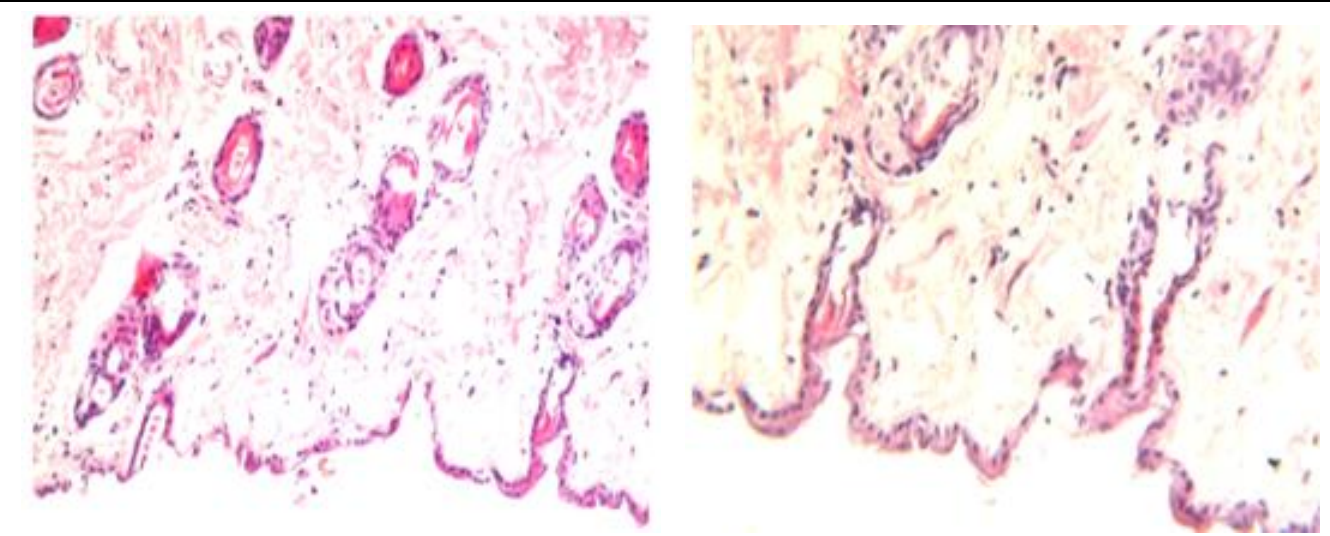

Figure7. Samples taken from the skin of recovery mice

\subsubsection{Eye}

Group (A): (mice kept as control)

There was no histopathological alteration and the normal histological structure of the cornea, iris and retina were recorded in Figure (8).

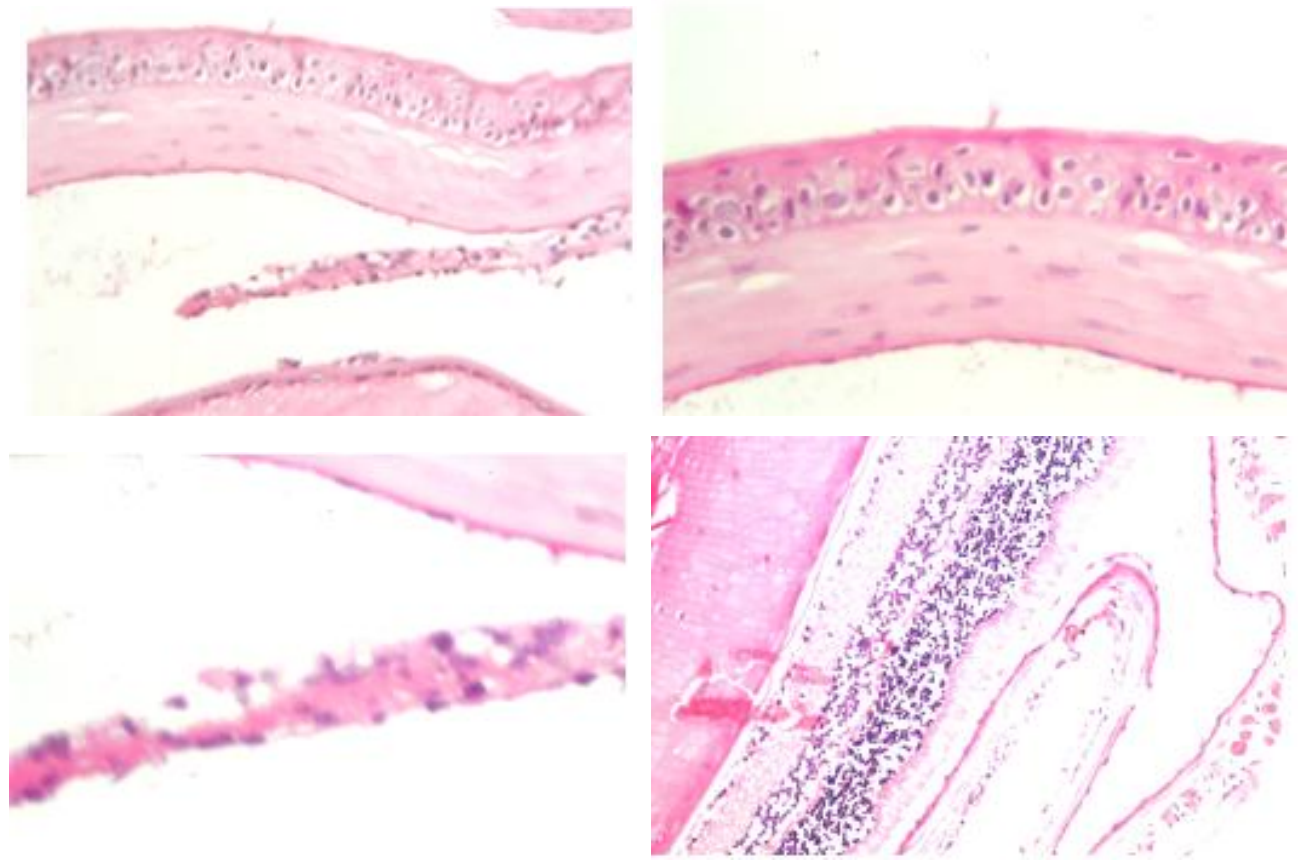

Figure8. Samples taken from the eye of mice kept as control

Group: (mice exposed to UV-C for one hour)

The endothelial cell layer of the cornea showed focal proliferation as shown in Figure (9).

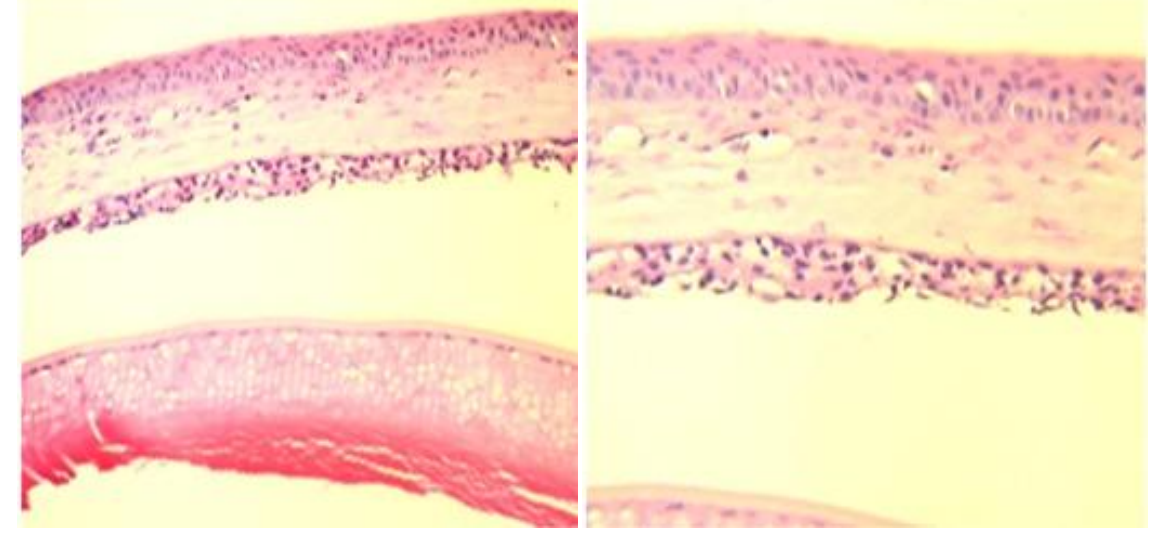

Figure9. Samples taken from the eye of mice exposed to $U V$-C for one hour 
Group (C): (Mice exposed to UV-C for two hour)

There were degeneration and necrosis in the covering epithelium of the cornea associated with destruction of the underlying basement membrane and stroma while the endothelial cell layer showed focal hyperplasia of the inner endothelial cell layer as shown in Figure (10).

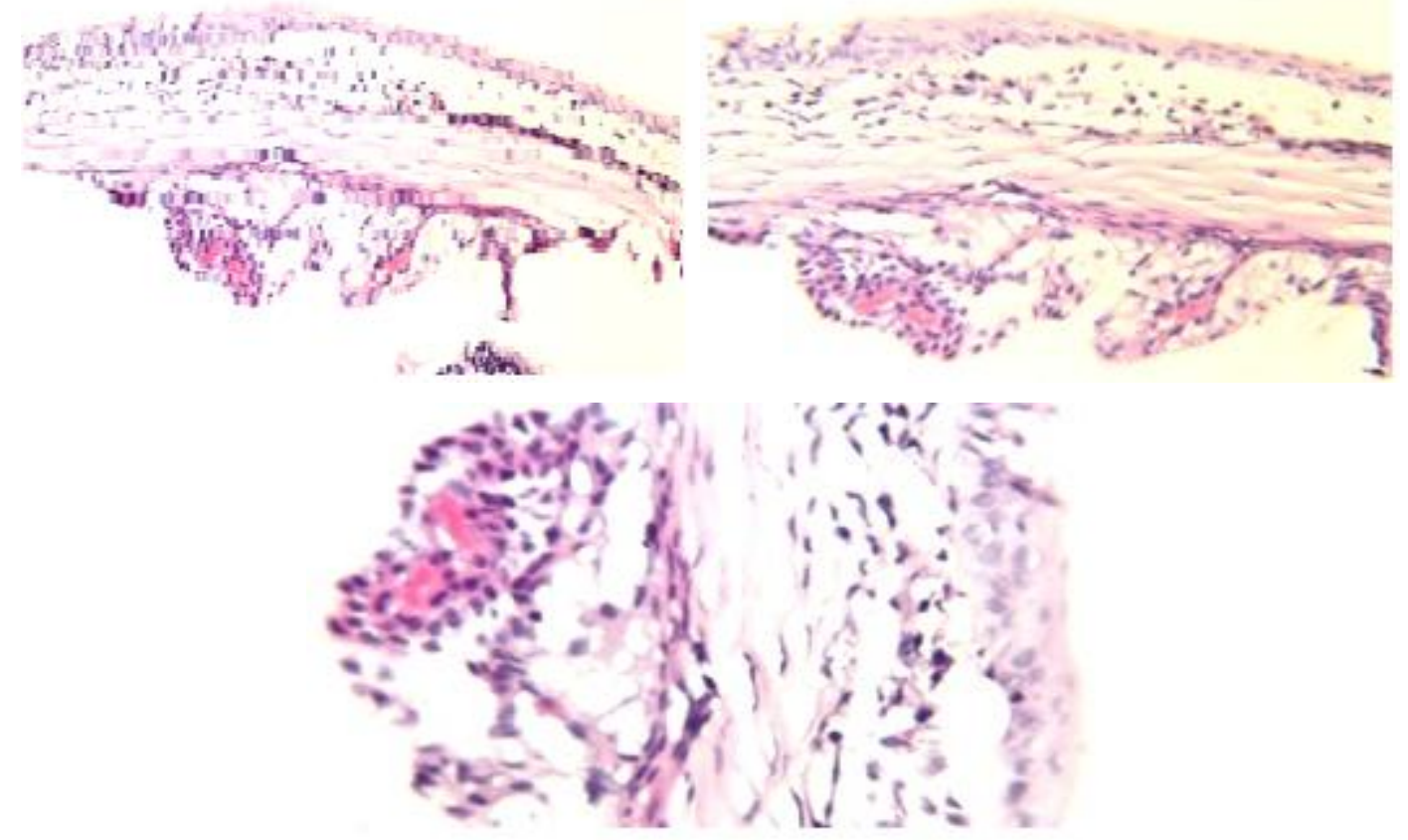

Figure10. Samples taken from the skin of mice exposed to UV-C for two hour

Group (D): (Recovery mice after exposer to UV-C for two hour)

Vacuolar degeneration was detected in the corneal epithelial layer as shown in Figure (11) while the endothelial corneal layer showed hyperplasia with polyps formation as shown in Figure (12).
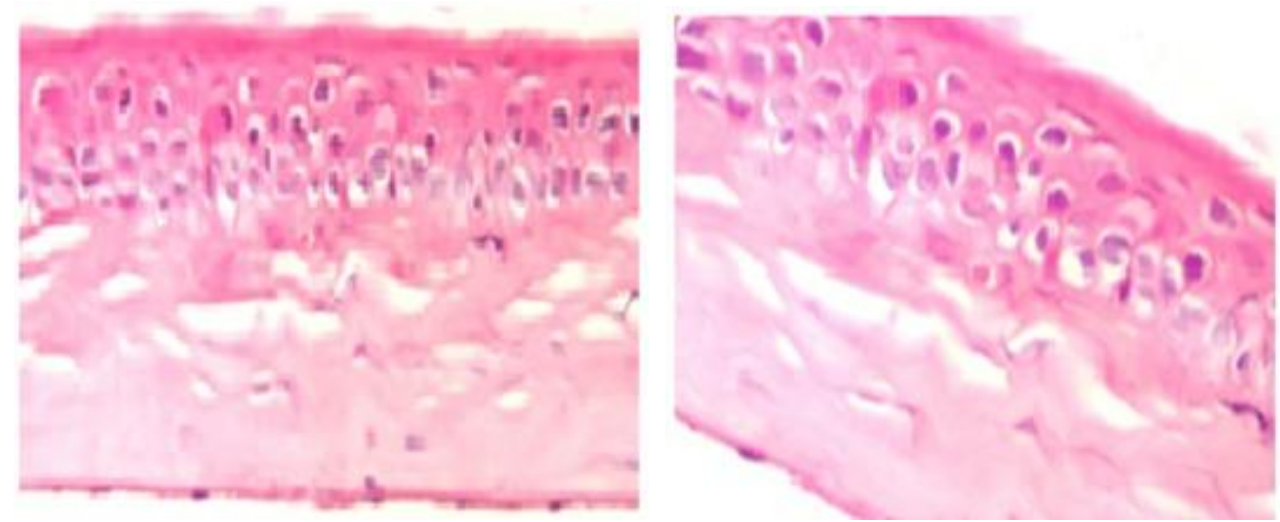

Figure11. Samples taken from the eye of recovery mice (the corneal epithelial layer)

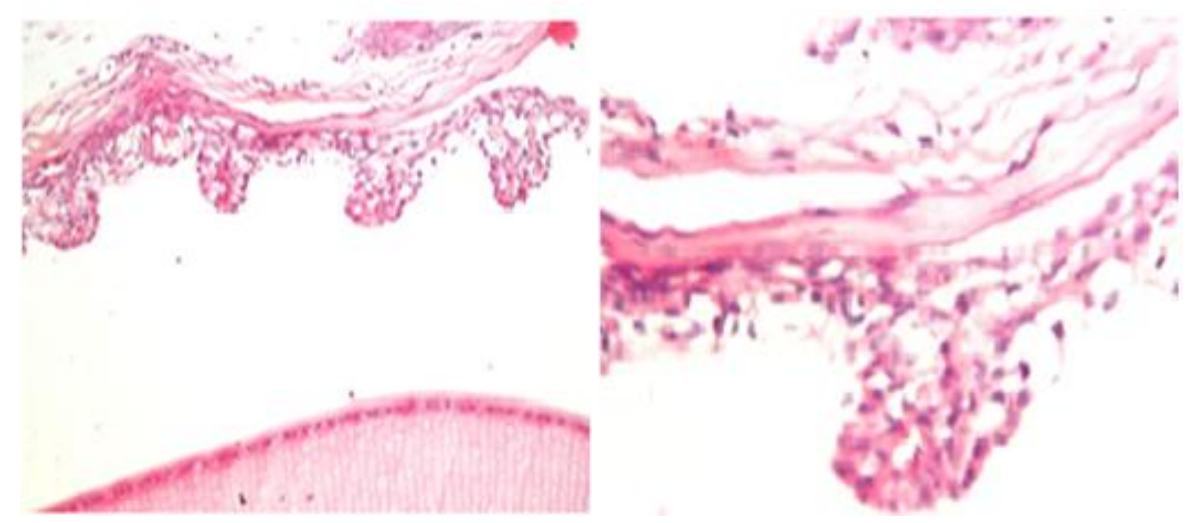

Figure12. Samples taken from the eye of recovery mice 


\section{Conclusions}

The objective of this work was to study the effect of ultraviolet-cradiation on the skin and eyes of mice. Three ultraviolet lamps with 15 watt for each, emit more than $85 \%$ of their energy in the UV-C ultraviolet radiation with a peak at $253.7 \mathrm{~nm}$ for germicidal action, were used in exposure. Autopsy samples were taken from the skin and eyes of mice in different groups and fixed in $10 \%$ formol saline for twenty four hours. Paraffin bees wax tissue blocks were prepared for sectioning at 4 microns thickness by slide microtome. The obtained tissue sections were collected on glass slides, deparaffinized, and stained by hematoxylin and eosin stain for examination through the light electric microscope.

In skin samples there was lose of the epidermal layers with intact basement membrane associated with invagination into the dermis. After recover the epidermal layer and the underlying dermis were intact while the invagination still present.

In eye samples there were degeneration and necrosis in the covering epithelium of the cornea associated with destruction of the underlying basement membrane and stroma while the endothelial cell layer showed focal hyperplasia of the inner endothelial cell layer. After recover vacuolar degeneration was detected in the corneal epithelial layer, while the endothelial corneal layer showed hyperplasia with polyps formation.

\section{REFERENCES}

[1] Icnirp Guide lines, Guide lines on limits of exposure to ultraviolet radiation of wavelength between $180 \mathrm{~nm}$ and $400 \mathrm{~nm}$ (Incoherent optical radiation), Health Physics 87: 2 (2004) 171-186

[2] Emory University, Environmental Health and Safety, Safety Toolbox Training- UV Radiation Facts, 2009

[3] Bergmanson J and Sheldon T. Ultraviolet radiation revisited. CLAO J23: (1997) 196-204

[4] Light Measurement Handbook () 1998 by Alex Ryer, International Light Inc.

[5] Banchroft, J.D., Stevens, A. And Turner, D.R. (1996). Theory and practice of histological techniques. Fourth Ed. Churchil Livingstone, New York, London, San Francisco, Tokyo.

[6] Havells Sylvania/ DataSheet (http://www.havells-sylvania.com/product/ro-ro/pdf/ DataSheet/ 0000502). 